descending (LAD). The full balloon was inflated and occluded the $\mathrm{LAD}$ for $60 \mathrm{~min}$ after ischaemia precondition in animals of group $\mathrm{A}$, and then the balloon embolism was positioned in the target vessel; but in group B, the balloon embolism was positioned in the target vessel directly. Intervention operation times and success rate were observed and compared with two groups.

Results 27 pigs underwent induction of AMI successful. Three pigs died of ventricular fibrillation and shock. Success rate of group A was $84.6 \%$, and that of group B was $94 \%$, there was not statistic significant compared with two groups. But mean operation time of group B $(28.4 \pm 9.4 \mathrm{~min})$ was shorter than that of group A (105.8 $\pm 27.6 \mathrm{~min})$, furthermore, compared with two groups, there was statistic significant.

Conclusions The method of establishment closed chest porcine model of AMI by implantation balloon embolism in target vessel is feasible, safe, quick and relatively effective.

\section{e0178 DIFFERENTIAL EXPRESSION OF N-MYC DOWNSTREAM REGULATED GENE 2 (NDRG2) IN THE RAT HEART AFTER ISCHAEMIA/REPERFUSION INJURY}

doi:10.1136/hrt.2010.208967.178

Zhongchan Sun, Haichang Wang, Guang Tong, Dongdong Sun, Feng Cao. Department of Cardiovascular medicine, Xijing Hospital, Fourth Military Medical University, Xi'an, China

Aims It has been shown that Ndrg2 (N-Myc downstream-regulated gene 2), a Myc-repressed gene, is markedly expressed in heart. Ndrg2 can act as a stress responsor under hypoxia and is necessary for hypoxia-induced apoptosis in certain tumour cell lines. In the present study, we investigated whether ischaemia/reperfusion (I/R) injury played a role in the regulation of $\mathrm{Ndrg} 2$ expression in rat heart and further explored the possible relationship between Ndrg2 expression and cardiomyocyte apoptosis induced by I/R injury.

Methods Rats were subjected to open chest surgery coronary artery ligation for ischaemia only or followed by reperfusion. Immunostaining and Western blot were applied to test the expression of Ndrg2, c-Myc, cleaved-caspase3 from myocardium, and TUNEL (terminal dUTP nick end labelling)-staining for apoptosis determination of myocardium.

Results The immunostaining confirmed Ndrg2 distribution in cardiomyocytes. The Ndrg2 expression in myocardial tissue after I/ $\mathrm{R}$ injury was significantly reduced at both $\mathrm{mRNA}$ and protein levels. We also observed that expression of c-Myc can be increased by I/R injury and was significantly inversely correlated with Ndrg2 expression. Furthermore, the rapid apoptotic rate at the early phase of reperfusion was ameliorated in the late phase. Some results in vivo were further confirmed by ex vivo study in cultured cardiomyocytes subjected to simulated I/R.

Conclusions Our data suggests that up-regulation of pro-apoptotic c-Myc expression induced by $\mathrm{I} / \mathrm{R}$ injury in rat myocardium may contribute to the down-regulation of also pro-apoptotic Ndrg2. Such stress response may be involved in the post I/R anti-apoptosis mechanism and myocardial repair in rat.

\section{e0179 IN ORDER TO INVESTIGATE THE POTENTIAL MECHANISM OF PIPERINE, WHICH IS THE ACTIVE SUBSTANCE FROM RHODOBRYUM ROSEUM LIMPR}

doi:10.1136/hrt.2010.208967.179

Kai REN, Zhao-liang SHAN. Department of Cardiology, Chinese PLA General Hospital, Beijing, China

Objectives In order to investigate the potential mechanism of Piperine, which is the active substance from Rhodobryum roseum
Limpr., on acute atrial electrical remodelling in atrial fibrillation by inducing of rapid atrial pacing, as well as its protective effect on injury of oxidative stress in myocardium.

Methods 24 healthy rabbits were collected, and randomly assigned to four groups as follows: normal saline (NS), normal saline+rapid atrial pacing (NS+RAP), piperine (PI), piperine+ rapid atrial pacing (PI+RAP). In the study, acute electrical remodelling was conducted by rapid atrial pacing. In pacing group, right atrium was paced with a frequency of $500-600 \mathrm{bpm}$ for $3 \mathrm{~h}$, atrial effective refractory period was measured at $0 \mathrm{~h}, 0.5 \mathrm{~h}, 1 \mathrm{~h}, 1.5 \mathrm{~h}, 2 \mathrm{~h}, 2.5 \mathrm{~h}$ and $3 \mathrm{~h}$ after pacing, respectively. Then we calculated the rate adaptation of atrial effective refractory periods in different basic pacing cycle lengths. Soon after the experiment, we dissected the atrium of rabbits, the left atrium, right atrium and pulmonary veins were dissected, consequently the levels of MDA, SOD, XOD and Calcium were measured with special kits. All the results were analysed with SPSS17.0.

Results 1. In the experiment, paroxysmal atrial fibrillation or atrial tachycardia can be induced only in NS+RAP group, whereas no similar phenomenon was observed in the other three groups. 2. AERP was markedly shorter in NS+RAP group but it was not changed in NS and PI+RAP group. The rate adaptation of AERP was reduced in NS+RAP, but got lowest point $(-0.24 \pm 0.59) 1 \mathrm{~h}$ after pacing, while the rate adaptation of AERP presented no significant changes in NS and PI group. 3. MDA of PI+RAP group in left atrium and pulmonary vein was lower than that of NS+RAP group $(p<0.01)$, but no significant difference of MDA in RA was observed between the two groups. 4. SOD activity in PV is higher in PI+RAP than that in NS+RAP, but no significant difference was observed in other locations between PI+RAP group and NS+RAP group 5. XOD activity in LA and PV is lower in PI+RAP than that in NS + RAP $(p<0.05)$, but XOD activity in RA presented no difference between the two groups. 6. Calcium level in LA, RA and PV, presented lower in PI+RAP compared with that in NS+RAP group. Conclusion 1. Piperine can help reduce incidence of AF, prevent the shortening of AERP and the rate adaptation of AERP, in other words, piperine can alleviate acute electrical remodelling in acute phase of AF. 2. Piperine can alleviate injury of oxidative stress in AF through suppression of MDA overproduction, reducing the consumption of SOD, suppression of XOD activity as well as Calcium overload, consequently develops the protective effect on myocardium during AF. 3. When AF is present, PV has the most serious injury of oxidative stress but RA suffer the slightest injury. Meanwhile, antioxidant effect of piperine is the most conspicuous in PV.

\section{e0180 THE ACUTE PROARRHYTHMIC EFFECTS OF LOW CONCENTRATION BPA ON FEMALE ADULT RAT AND THE ELECTROPHYSIOLOGIC MECHANISIMS}

doi:10.1136/hrt.2010.208967.180

${ }^{1}$ Sujuan Yan, ${ }^{1}$ Xiaoshu Cheng, ${ }^{1}$ Kui Hong, ${ }^{2}$ Weizhong Song, ${ }^{2}$ Yamei Chen. ${ }^{1}$ Department of Cardiology, The Second Affiliated Hospital of Nanchang University, Nanchang, Jiangxi, China; ${ }^{2}$ Department of Pharmacology, College of Medicine, University of Cincinnati, Cincinnati, Ohio, USA

Objective To investigate the acute proarrhythmic effects of low concentration BPA on adult rat and to demonstrate the electrophysiologic mechanisms.

Methods and results Acute exposure to BPA increased the contractility of cardiac myocytes from female rat heart with inverted U-shaped dose-response curve, these effects were female specific. After-contraction or after-transient rate of female rat cardiac myocytes increased in BPA group, and increased much more by exposure to the mixture of BPA and $10^{-9} \mathrm{M} \mathrm{E}_{2}$. Increasing BPA or $\mathrm{E}_{2}$ from $10^{-9} \mathrm{M}$ to $2 \mathrm{X} 10^{-9} \mathrm{M}$ did not increase the effects induced responses. Although BPA combined with E2 did not induce the 